\title{
Quasi-two-dimensional Feynman bipolarons
}

\author{
R. T. Senger and A. Erçelebi \\ Department of Physics, Bilkent University, 06533 Ankara, Turkey
}

(Received 15 January 1999)

\begin{abstract}
We study the stability criterion for the formation of two-dimensionally confined large bipolarons. The electrons are treated as bounded within a parabolic potential well while being coupled to one another via the Fröhlich interaction Hamiltonian. Within the framework of the bulk-phonon approximation we adopt the Feynman-polaron model to derive variational results over a wide range of the Coulomb interaction and phonon coupling strengths interpolating between the bulk and the two-dimensional confinement limit. It is shown that the critical values of the electron-phonon coupling constant and the ratio of dielectric constants $\left(\eta=\epsilon_{\infty} / \epsilon_{0}\right)$ exhibit some nontrivial features as the effective dimensionality is tuned from 3 to 2. [S0163-1829(99)08637-3]
\end{abstract}

\section{INTRODUCTION}

Two electrons in a polar or ionic crystal interact with the lattice vibrations resulting in attractive forces between them. Under certain conditions, the phonon mediated attraction between the particles may come out to be strong enough to counterbalance the Coulomb repulsion and consequently, a stable bound state can form. Such a state of the system consisting of the pair of electrons and a common density of virtual phonons is termed a bipolaron.

Among the numerous amount of papers published within the context of two-polaron systems, we at first cite the pioneering work of Haken ${ }^{1}$ who studied the problem of the interaction between an electron and a hole via the coupling to the LO branch of the phonon spectrum in polar semiconductors. Using a variational method he showed that the polaron corrections to the effective interaction at large distances decrease exponentially, reflecting the exponentially decreasing overlap between the clouds of bound charges around the polarons. The same problem was further considered by Mahanti and $\mathrm{Varma}^{2}$ and by $\mathrm{Sak}^{3}$ where they included the corrections to the electron-hole potential coming from the dynamic polarization of the lattice, and showed how deviations from the Coulomb form could occur. The intrinsic effect of electron (hole) phonon interactions on the nature of forces acting between the particles was reconsidered by Kuleshov, Matveev, and Smondyrev ${ }^{4}$ and the same work was revised later. ${ }^{5}$ Basing their calculations on Feynman path-integral formalism, they developed a scheme for obtaining expressions for the particle interaction potentials and the ground state energy in both the strong- and weak-coupling approximations. A similar problem in the same area was considered by Bishop and Overhauser $^{6}$ to investigate the phonon-mediated interaction between two electrons where they showed that for ionic crystals the effective electron-electron potential may lead to an attractive deep potential well with a minimum occurring for particle separations as small as a few tens of $\AA$.

In the bipolaron problem, with the electrons being closely positioned, the polarization fields centered about the particles overlap and interfere in a constructive manner to create a potential well deep enough to compete with the Coulomb repulsion so as to prevent the particles from being projected apart. Depending on the dielectric properties of the medium, provided the effective Coulomb repulsion is not larger than a critical strength and the phonon coupling constant is not smaller than a critical value, the lattice effects may account for a considerable part of the energy of the electron-electron pair which consists of attracting the particles against their Coulomb repulsion. Thus, the fundamental condition under which a bipolaron can exist is that the repulsive Coulomb interaction should not be too strong to dominate over and hence break up the phonon-mediated binding which holds the particles together.

In the last two decades, the study of bipolarons has attracted a revived and extensive interest in the literature. ${ }^{7-26}$ Of particular relevance to the content of the present article are the recent solutions of the bipolaron problem in three and strict two dimensions ${ }^{14-16,21}$ where it is observed that a bipolaronic bound state of two electrons is more easily attained in two dimensions than in three. The concern of the present study is to extend the problem to a broader discourse and explore the stability of quasi-two-dimensional bipolarons confined in a parabolic quantum well with variable well width and potential barrier slopes; thus provide an interpolating insight into the phase diagram, encompassing the bulk and the two-dimensional limits. The harmonic-oscillator confining potential that we adopt here has already been used in a parallel study ${ }^{26}$ within the framework of the strong-coupling polaron theory where it has been noted that, in the quasi-twodimensional (slablike) configuration, the utmost permissible Coulomb strength which would allow a bipolaron state to form may turn out to be lower than one would have for the three- and strict two-dimensional bipolarons. To see whether this peculiar feature is indeed a characteristic of quasi-twodimensionally confined bipolarons, we wish to review the problem within a similar framework of the three and twodimensional bipolaron models set up earlier by Verbist, Peeters, and Devreese ${ }^{14}$ where they reformulate the Feynman path-integral variational approach ${ }^{27}$ to tackle the case of two interacting polarons. Thus, a critical understanding of the foregoing calculations and the outcomes depends heavily on having read Ref. 14 with which we shall make some frequent correspondence, particularly when the three- and twodimensional limits of our model are concerned. We believe, the methodology followed in this work proves to be a powerful technique intended to yield a satisfactory description of 
the problem in the overall ranges of the parameters characterizing the system. In the following, we obtain an explicit tracking of the phase stability in terms of the Coulomb and phonon-coupling strengths as a function of the effective dimensionality tuned from 3 to 2 .

\section{THEORY}

\section{A. Bipolaron model}

The model that we use consists of a pair of quasi-twodimensional electrons coupled to the LO branch of the bulk phonon spectrum. As such, the fundamental approach followed in this work is to account for mainly the generic lowdimensional aspect of the dynamical behavior of the electrons and visualize them as interacting with the medium and with one another through exhange of virtual phonons. Apart from ignoring the contributions that may come from all other kinds of phonon modes, we also omit the screening effects and further details, such as those due to the nonparabolicity corrections to the electron band or the loss of validity of both the effective-mass approximation and the Fröhlich continuum Hamiltonian in microstructures. Hence, under the socalled bulk-phonon approximation and the aforementioned simplifying assumptions, we write the Hamiltonian describing the confined electron-pair system coupled to the LO phonon field as

$$
\mathrm{H}=H_{e}+\sum_{Q} a_{Q}^{\dagger} a_{Q}+\sum_{i=1,2} \sum_{Q} V_{Q}\left(a_{Q} e^{i \vec{Q} \cdot \vec{r}_{i}}+a_{Q}^{\dagger} e^{-i \vec{Q} \cdot \vec{r}_{i}}\right),
$$

where

$$
H_{e}=\sum_{i=1,2}\left(\frac{1}{2} p_{i}^{2}+V_{\mathrm{conf}}\left(z_{i}\right)\right)+\frac{U}{\left|\vec{r}_{1}-\vec{r}_{2}\right|} .
$$

Here and henceforth we use dimensionless units appropriate to a polaron calculation and take $m^{*}=\hbar=\omega_{L O}=1$. In Eqs. (1), (2), $a_{Q}$ and $a_{Q}^{\dagger}$ denote the phonon annihilation and creation operators, and $\vec{r}_{i}=\left(\vec{\rho}_{i}, z_{i}\right) \quad(i=1,2)$, are the positions of the electrons in cylindrical coordinates. Similarly, $\vec{p}_{i}(i$ $=1,2$ ) denote the respective momenta of the electrons. The Fröhlich interaction amplitude is related to the phonon wave vector $\vec{Q}=\left(\vec{q}, q_{z}\right)$ through

$$
V_{Q}=(2 \sqrt{2} \pi \alpha)^{1 / 2}|\vec{Q}|^{-1},
$$

where $\alpha$ is the coupling constant defined, in terms of the high frequency and static dielectric constants of the medium, by

$$
\alpha=\frac{e^{2}}{\sqrt{2}}\left(\frac{1}{\epsilon_{\infty}}-\frac{1}{\epsilon_{0}}\right) .
$$

In the Coulomb term, the unscreened amplitude $U$ is related to the ratio of the dielectric constants

$$
\eta=\frac{\epsilon_{\infty}}{\epsilon_{0}}<1
$$

through

$$
U=\frac{e^{2}}{\epsilon_{\infty}}=\frac{\sqrt{2}}{1-\eta} \alpha .
$$

For the confining potential we use a harmonic oscillator profile with adjustable barrier slopes, i.e., we set

$$
V_{\text {conf }}(z)=\frac{1}{2} \Omega^{2} z^{2}
$$

in which the dimensionless frequency $\Omega$ serves for the measure of the degree of confinement of the electron which, when tuned from zero to infinity, yields a unifying display of the phase stability of the bipolaron as a function of the effective dimensionality ranging from 3 to 2 . The rationale behind imposing quadratic potential profiles is that such a form for the confining barriers greatly facilitates the calculations and leads to tractable analytic expressions. We have thus refrained ourselves from treating potentials of other forms which possibly would lead to complicated and even prohibitively difficult expressions and numerical complications and yet yield qualitative features similar to those for parabolic potential shapes. Indeed, calculations pertaining to the cyclotron study of polarons confined to an interface indicate that the phonon-coupling-induced shift in the resonant energy is sensitive dominantly to the strength of the confining potential rather than its shape. ${ }^{28}$ Moreover, due to the absence of an abrupt variation in the medium structure and properties, the parabolic confining potential allows us to disregard any relevance to the interface phonon modes. ${ }^{29} \mathrm{We}$, therefore, conveniently use the harmonic-oscillator potential (6) as a simplifying first approximation compatible with the framework of the path integral approach where one assumes the two electrons to be coupled to one another and to the corresponding fictitious particles via harmonic springs.

In the Feynman path integral representation of the polaron, the phonon variables can be projected out exactly to yield the partition function of the bipolaron system in the form

$$
\mathcal{Z}_{\mathrm{BP}}=\mathcal{Z}_{\mathrm{ph}} \mathcal{Z}
$$

where

$$
\mathcal{Z}_{\mathrm{ph}}=\prod_{Q}\left(\frac{1}{1-e^{-\beta \hbar \omega_{\mathrm{LO}}}}\right)^{3}
$$

is the phonon part, and

$$
\mathcal{Z}=\prod_{i=1,2}\left(\int d \vec{r}_{0} \int_{\vec{r}_{i}(0)=\vec{r}_{0}}^{\vec{r}_{i}(\beta)=\vec{r}_{0}} \mathcal{D} \vec{r}_{i}(\lambda)\right) e^{\mathcal{S}\left[\vec{r}_{1}(\lambda), \vec{r}_{2}(\lambda)\right]}
$$

is the path integral in which the action $\mathcal{S}$ consists of two parts, one pertaining to the electron part of the Hamiltonian and the other to the electron-phonon interaction. In imaginary time variables $(t \rightarrow-i \lambda)$, we have respectively, the following expressions:

$$
\mathcal{S}_{e}=-\frac{1}{2} \int_{0}^{\beta} d \lambda \sum_{i=1,2}\left[\dot{\vec{r}}_{i}^{2}(\lambda)+\Omega^{2} z_{i}^{2}(\lambda)\right]+\mathcal{S}_{c},
$$

where 


$$
\mathcal{S}_{c}=-\int_{0}^{\beta} d \lambda \frac{U}{\left|\vec{r}_{1}(\lambda)-\vec{r}_{2}(\lambda)\right|}
$$

is the Coulomb term, and

$$
\begin{aligned}
\mathcal{S}_{e-p}= & \frac{1}{2} \sum_{i=1,2} \sum_{j=1,2} \sum_{Q} V_{Q}^{2} \int_{0}^{\beta} d \lambda \int_{0}^{\beta} d \lambda^{\prime} G_{\left(\omega_{\mathrm{LO}}=1\right)}\left(\lambda-\lambda^{\prime}\right) \\
& \times e^{i \vec{Q} \cdot\left[\vec{r}_{i}(\lambda)-\vec{r}_{j}\left(\lambda^{\prime}\right)\right]}
\end{aligned}
$$

is the part describing the phonon mediated retarded attractive interaction between the electrons. In the above, the dimensionless parameter $\beta$ stands for the inverse temperature, and the memory function

$$
G_{\omega}(u)=\frac{\cosh [(\beta-2|u|) \omega / 2]}{\sinh (\beta \omega / 2)}
$$

is the Green's function of a harmonic oscillator with frequency $\omega$. In principle, at zero temperature, we have $\mathcal{Z}_{\mathrm{BP}}$ $=\mathcal{Z}$, and the bipolaron ground state energy

$$
E_{g}=-\lim _{\beta \rightarrow \infty} \beta^{-1} \log \mathcal{Z}
$$

can be calculated exactly provided the path integral (9) can be evaluated. Since this is not possible due to the analytic complexity of the integral expressions in the action $\mathcal{S}$, Eqs. (10)-(12), we choose to proceed with the introduction of a solvable trial action $\mathcal{S}_{0}$ intended to provide us with a convenient variational upper bound to the ground state energy, led by the Jensen-Feynman inequality

$$
E_{g} \leqslant E_{0}-\lim _{\beta \rightarrow \infty} \frac{1}{\beta}\left\langle\mathcal{S}-\mathcal{S}_{0}\right\rangle_{\mathcal{S}_{0}},
$$

where the notation \langle\rangle$_{\mathcal{S}_{0}}$ denotes a path-integral average with density function $e^{\mathcal{S}_{0}}$, and $E_{0}$ is the trial ground state energy corresponding to $\mathcal{S}_{0}$. The form of the trial action should be simple enough to permit analytical calculations and yet be detailed enough to cover all the basic features of the exact action $\mathcal{S}$.

\section{B. The trial model and its diagonalization}

For the trial action we choose the model which was successfully applied to similar polaron or bipolaron problems ${ }^{14,27,30}$ where the electrons are considered to be in quadratic interaction with the fictitious masses. We write $\mathcal{S}_{0}$ as a sum of three terms, i.e.,

$$
\mathcal{S}_{0}=\mathcal{S}_{e}+\mathcal{S}_{s}+\mathcal{S}_{m}
$$

where $\mathcal{S}_{e}$ is similar to that given by Eq. (10), except now that the Coulomb term (11) is reexpressed as

$$
\mathcal{S}_{c}=\frac{1}{2} K \int_{0}^{\beta} d \lambda\left[\vec{r}_{1}(\lambda)-\vec{r}_{2}(\lambda)-\vec{a}\right]^{2}
$$

$\mathcal{S}_{s}$ and $\mathcal{S}_{m}$ refer to the self- and mutual interaction of the electrons with the fictitious masses, each with its own and with that of the other electron, respectively. These terms assume the following path-integral representations

$$
\mathcal{S}_{s}=-\frac{c_{\mathrm{s}}}{2} \int_{0}^{\beta} d \lambda \int_{0}^{\beta} d \lambda^{\prime} G_{\mathrm{w}}\left(\lambda-\lambda^{\prime}\right) \sum_{i=1,2}\left[\vec{r}_{i}(\lambda)-\vec{r}_{i}\left(\lambda^{\prime}\right)\right]^{2},
$$

$$
\begin{aligned}
\mathcal{S}_{m}= & -2 \times \frac{c_{\mathrm{m}}}{2} \int_{0}^{\beta} d \lambda \int_{0}^{\beta} d \lambda^{\prime} G_{\mathrm{w}}\left(\lambda-\lambda^{\prime}\right) \\
& \times\left[\vec{r}_{1}(\lambda)-\vec{r}_{2}\left(\lambda^{\prime}\right)-\vec{a}\right]^{2} .
\end{aligned}
$$

In the above $K, c_{\mathrm{s}}, c_{\mathrm{m}}$ and $\mathrm{w}$ are variational parameters introduced within a similar context as in the original paper by Feynman. ${ }^{27}$ Vector $\vec{a}$ is a further parameter describing the mean distance between the central positions about which the electrons fluctuate. We should note that the confining potential (6), being symmetric in the $\pm z$ directions, imposes the mean positions of both electrons to lie in the $x y$ plane. We therefore readily set $a_{z}=0$. We should also remark that in the extreme limits $\vec{a}=0$ and $|\vec{a}| \rightarrow \infty$, the model yields respectively a description of either the bipolaronic state of the two electrons or a pair of two independent polarons. ${ }^{14}$

Since the trial action and the path-integral averages involved in Eqs. (14)-(18) are all separable in the Cartesian coordinates, the calculations can be performed all in identical manners for each spatial direction. Denoting the Cartesian component of the $i$ th electron in any chosen direction by $x_{i}$ and that of the $i$ th fictitious mass $M$ by $X_{i}$, the part of the model Lagrangian relevant to that coordinate

$$
\begin{aligned}
L_{\text {mod }}^{(x)}= & \frac{1}{2} \sum_{i=1,2}\left(\dot{x}_{i}^{2}-\Omega^{2} x_{i}^{2}+M \dot{X}_{i}^{2}\right)+\frac{K}{2}\left(x_{1}-x_{2}-a_{x}\right)^{2} \\
& -\frac{\kappa}{2} \sum_{i=1,2}\left(x_{i}-X_{i}\right)^{2} \\
& -\frac{\kappa^{\prime}}{2}\left\{\left(x_{1}-X_{2}-a_{x}\right)^{2}+\left(x_{2}-X_{1}+a_{x}\right)^{2}\right\}
\end{aligned}
$$

can be related to the following model action:

$$
\begin{aligned}
\mathcal{S}_{\bmod }^{(x)}= & -\frac{1}{2} \int_{0}^{\beta} d \lambda \sum_{i=1,2}\left[\dot{x}_{i}^{2}(\lambda)+\Omega^{2} x_{i}^{2}(\lambda)+M \dot{X}_{i}^{2}(\lambda)\right] \\
& +\frac{1}{2} \int_{0}^{\beta} d \lambda\left\{K\left[x_{1}(\lambda)-x_{2}(\lambda)-a_{x}\right]^{2}\right. \\
& \left.-\sum_{i=1,2} \kappa\left[x_{i}(\lambda)-X_{i}(\lambda)\right]^{2}\right\} \\
& -\frac{\kappa^{\prime}}{2} \int_{0}^{\beta} d \lambda\left\{\left[x_{1}(\lambda)-X_{2}(\lambda)-a_{x}\right]^{2}\right. \\
& \left.+\left[x_{2}(\lambda)-X_{1}(\lambda)+a_{x}\right]^{2}\right\} .
\end{aligned}
$$

Here, it should be understood that $\Omega$ has its original meaning as in Eq. (10) when one refers to the $z$ direction, but assumes zero value for the remaining two directions.

Similar to the elimination of the phonon degrees of freedom, also eliminating the fictitious mass coordinates $X_{i}$, we obtain the relevant trial action, expressed solely in terms of the electron coordinates, in the form as given in Eqs. (15)- 
(18). The variational parameters of the trial action can then be identified in terms of the parameters of the model Lagrangian

$$
w=\sqrt{\frac{\kappa+\kappa^{\prime}}{M}}, \quad c_{s}=\frac{\kappa^{2}+\kappa^{\prime 2}}{4 M w}, \quad c_{m}=\frac{\kappa \kappa^{\prime}}{2 M w} .
$$

In order to calculate the ground state energy of the variational model and the path integral average required in Eq. (14), one needs to diagonalize $L_{\text {mod }}^{(x)}$ (19). Introducing $x_{\text {c.m. }}\left(X_{\text {c.m. }}\right)$ and $x_{\text {rel }}\left(X_{\text {rel }}\right)$ as the center of mass and relative coordinates for the electrons (fictitious masses) through

$$
\begin{aligned}
& \left\{\begin{array}{l}
x_{1} \\
x_{2}
\end{array}\right\}=\frac{1}{2}\left(x_{\text {c.m. }} \pm x_{\text {rel }}\right) \pm \frac{a_{x}}{2}, \\
& \left\{\begin{array}{l}
X_{1} \\
X_{2}
\end{array}\right\}=\frac{1}{2}\left(X_{\text {c.m. }} \pm X_{\text {rel }}\right) \pm \frac{a_{x}}{2}
\end{aligned}
$$

we rewrite the Lagrangian relevant to the chosen direction as

$$
L_{\text {mod }}^{(x)}=L_{\text {c.m. }}^{(x)}+L_{\text {rel }}^{(x)},
$$

where

$$
\begin{aligned}
L_{\mathrm{c} . \mathrm{m} .}^{(x)}= & \frac{1}{4} \dot{x}_{\mathrm{c} . \mathrm{m} .}^{2}+\frac{1}{4} M \dot{X}_{\mathrm{c} . \mathrm{m} .}^{2}-\frac{1}{4}\left(\Omega^{2}+\kappa+\kappa^{\prime}\right) x_{\mathrm{c} . \mathrm{m} .}^{2} \\
& -\frac{1}{4}\left(\kappa+\kappa^{\prime}\right) X_{\mathrm{c} . \mathrm{m} .}^{2}+\frac{1}{2}\left(\kappa+\kappa^{\prime}\right) x_{\mathrm{c} . \mathrm{m} .} X_{\mathrm{c} . \mathrm{m} .}, \\
L_{\mathrm{rel}}^{(x)}= & \frac{1}{4} \dot{x}_{\mathrm{rel}}^{2}+\frac{1}{4} M \dot{X}_{\mathrm{rel}}^{2}-\frac{1}{4}\left(\Omega^{2}+2 K+\kappa+\kappa^{\prime}\right) x_{\mathrm{rel}}^{2} \\
& -\frac{1}{4}\left(\kappa+\kappa^{\prime}\right) X_{\mathrm{rel}}^{2}+\frac{1}{4}\left(\kappa-\kappa^{\prime}\right) x_{\mathrm{rel}} X_{\mathrm{rel}} .
\end{aligned}
$$

We should note that we have excluded a final term $-\frac{1}{2} \Omega^{2} a_{x} x_{\text {rel }}$ which has had to appear on the right hand side of Eq. (23), since this term always vanishes because, either $\Omega$ or $a_{x}$ is zero for all Cartesian directions.

Under appropriate coordinate transformations

$$
\begin{gathered}
x_{\mathrm{c} . \mathrm{m} .}=\eta_{0}+\eta_{1}, \\
X_{\mathrm{c} . \mathrm{m} .}=\frac{w^{2}}{w^{2}-\xi_{0}^{2}} \eta_{0}+\frac{w^{2}}{w^{2}-\xi_{1}^{2}} \eta_{1}, \\
x_{\text {rel }}=\eta_{2}+\eta_{3}, \\
X_{\mathrm{rel}}=\frac{\kappa-\kappa^{\prime}}{\kappa+\kappa^{\prime}} \frac{w^{2}}{w^{2}-\xi_{2}^{2}} \eta_{2}+\frac{\kappa-\kappa^{\prime}}{\kappa+\kappa^{\prime}} \frac{w^{2}}{w^{2}-\xi_{3}^{2}} \eta_{3} .
\end{gathered}
$$

$L_{\mathrm{c} . \mathrm{m} .}^{(x)}$ and $L_{\mathrm{rel}}^{(x)}$ can be diagonalized in the respective normal coordinates $\left\{\eta_{0}, \eta_{1}\right\}$ and $\left\{\eta_{2}, \eta_{3}\right\}$, yielding the canonical forms

$$
L_{\mathrm{c} . \mathrm{m} .}^{(x)}=\frac{1}{2} \sum_{i=0,1} m_{i}\left(\dot{\eta}_{i}^{2}-\xi_{i}^{2} \eta_{i}^{2}\right),
$$

$$
L_{\mathrm{rel}}^{(x)}=\frac{1}{2} \sum_{i=2,3} m_{i}\left(\dot{\eta}_{i}^{2}-\xi_{i}^{2} \eta_{i}^{2}\right)
$$

where

$$
\begin{gathered}
m_{0}=\frac{1}{2} \frac{\xi_{1}^{2}-\xi_{0}^{2}}{w^{2}-\xi_{0}^{2}}, \quad m_{1}=\frac{1}{2} \frac{\xi_{1}^{2}-\xi_{0}^{2}}{\xi_{1}^{2}-w^{2}}, \\
m_{2}=\frac{1}{2} \frac{\xi_{2}^{2}-\xi_{3}^{2}}{\xi_{2}^{2}-w^{2}}, \quad m_{3}=\frac{1}{2} \frac{\xi_{2}^{2}-\xi_{3}^{2}}{w^{2}-\xi_{3}^{2}}
\end{gathered}
$$

are the relevant mass values and $\xi_{i}(\Omega)(i=0,1,2,3)$ refer to the eigenfrequencies, given by

$$
\begin{aligned}
\left\{\begin{array}{c}
\xi_{0} \\
\xi_{1}
\end{array}\right\}= & \frac{1}{\sqrt{2}}\left\{\Omega^{2}+\omega_{1}^{2} \mp \sqrt{\left(\Omega^{2}+\omega_{1}^{2}\right)^{2}-4 \Omega^{2} w^{2}}\right\}^{1 / 2}, \\
\left\{\begin{array}{c}
\xi_{2} \\
\xi_{3}
\end{array}\right\}= & \frac{1}{\sqrt{2}}\left\{\Omega^{2}+\omega_{2}^{2}+\omega_{3}^{2}\right. \\
& \left. \pm \sqrt{\left(\Omega^{2}+\omega_{2}^{2}+\omega_{3}^{2}\right)^{2}-4\left(\Omega^{2} w^{2}+\omega_{2}^{2} \omega_{3}^{2}\right)}\right\}^{1 / 2} .
\end{aligned}
$$

We note that, in the limit $\Omega \rightarrow 0$, the eigenfrequencies $\xi_{i}$ reduce to

$$
\xi_{0}(0)=0 \text { and } \xi_{i}(0)=\omega_{i} \quad(i=1,2,3)
$$

in which $\omega_{i}$ are the normal mode frequencies calculated previously by Verbist, Peeters, and Devreese ${ }^{14}$ for the bulk case, expressed as

$$
\omega_{1}=\left\{\frac{M+1}{M}\left(\kappa+\kappa^{\prime}\right)\right\}^{1 / 2},
$$

$$
\begin{aligned}
\left\{\begin{array}{c}
\omega_{2} \\
\omega_{3}
\end{array}\right\}= & \frac{1}{\sqrt{2}}\left\{\frac{M+1}{M}\left(\kappa+\kappa^{\prime}\right)-2 K\right. \\
& \left. \pm \sqrt{\left[\frac{M-1}{M}\left(\kappa+\kappa^{\prime}\right)-2 K\right]^{2}+\frac{4}{M}\left(\kappa-\kappa^{\prime}\right)^{2}}\right\}^{1 / 2} .
\end{aligned}
$$

Here we shall adopt the conventional HO (harmonic oscillator) operator representation which allows us to calculate all the required path integral averages easily. On this purpose we introduce the lowering and raising operators $c_{i}$ and $c_{i}^{\dagger}$, $(i=0,1,2,3)$, defined by

$$
\eta_{i}=\lambda_{i}\left(c_{i}^{\dagger}+c_{i}\right), \quad\left[c_{i}, c_{i}^{\dagger}\right]=1
$$

in which

$$
\lambda_{i}=\left(2 m_{i} \xi_{i}\right)^{-1 / 2}
$$

The corresponding Hamiltonian relevant to $L_{\bmod }^{(x)}$ can be expressed in the $\mathrm{HO}$ form

$$
H_{\mathrm{mod}}^{(x)}=\sum_{i=0}^{3} \xi_{i}(\Omega)\left(c_{i}^{\dagger} c_{i}+\frac{1}{2}\right)
$$


where the summation index $i$ takes values 0 and 1 for the center of mass coordinates, and values 2 and 3 for the relative coordinates. Hence, the part of the ground-state energy contributed by the particular coordinate relevant to $\mathcal{S}_{0}^{(x)}$ is obtained simply as

$$
E_{0}^{(x)}=-w+\frac{1}{2} \sum_{i=0}^{3} \xi_{i}(\Omega),
$$

wherein the additional term $w$ comes about under eliminating the fictitious mass coordinates to obtain the trial action $\mathcal{S}_{0}$ from the model action.

Noting that the confining parameter $\Omega$ is relevant only to the $z$ axis, and that it has to be accounted for having value zero in the remaining two directions, the expression for $E_{0}^{(x)}$ can be extended to account for each of the Cartesian coordinates all at once, yielding

$$
E_{0}=-3 w+\sum_{i=0}^{3}\left[\xi_{i}(0)+\frac{1}{2} \xi_{i}(\Omega)\right] .
$$

\section{Path-integral averages}

In order to reach the upper bound to the bipolaronic ground state energy, one has to evaluate the path integral average involved in Eq. (14), where

$$
\begin{aligned}
\left\langle\mathcal{S}-\mathcal{S}_{0}\right\rangle_{\mathcal{S}_{0}}= & -\frac{K}{2} \int_{0}^{\beta} d \lambda\left\langle\left[\vec{r}_{1}(\lambda)-\vec{r}_{2}(\lambda)-\vec{a}\right]^{2}\right\rangle_{\mathcal{S}_{0}} \\
& -U \int_{0}^{\beta} d \lambda \sum_{Q} \frac{4 \pi}{Q^{2}}\left\langle e^{i \vec{Q} \cdot\left[\vec{r}_{1}(\lambda)-\vec{r}_{2}(\lambda)\right]}\right\rangle_{\mathcal{S}_{0}} \\
& +\frac{1}{2} \int_{0}^{\beta} d \lambda \int_{0}^{\beta} d \lambda^{\prime} G_{w}\left(\lambda-\lambda^{\prime}\right) \\
& \times\left\{c _ { \mathrm { s } } \sum _ { i = 1 , 2 } \left\langle\left[\vec{r}_{i}\left(\lambda-\vec{r}_{i}\left(\lambda^{\prime}\right)\right]^{2}\right\rangle_{\mathcal{S}_{0}}\right.\right. \\
& \left.+2 c_{m}\left\langle\left[\vec{r}_{1}(\lambda)-\vec{r}_{2}\left(\lambda^{\prime}\right)-\vec{a}\right]^{2}\right\rangle_{\mathcal{S}_{0}}\right\} \\
& +\frac{1}{2} \sum_{Q} V_{Q}^{2} \int_{0}^{\beta} d \lambda \int_{0}^{\beta} d \lambda^{\prime} G_{\omega_{\mathrm{LO}}}\left(\lambda-\lambda^{\prime}\right) \\
& \times \sum_{i, j=1,2}\left\langle e^{i \vec{Q} \cdot\left[\vec{r}_{i}(\lambda)-\vec{r}_{j}\left(\lambda^{\prime}\right)\right]}\right\rangle_{\mathcal{S}_{0}}
\end{aligned}
$$

in which, for computational convenience, the Coulomb potential has been written in its Fourier expanded form.

Using the well established identity for a harmonic oscillator with frequency $\omega$ and the relevant annihilation and creation operators $c$ and $c^{\dagger}$

$$
\begin{aligned}
& \left\langle\exp \left\{\int_{0}^{\beta} d \lambda\left[f^{*}(\lambda) c^{\dagger}+f(\lambda) c\right]\right\}\right\rangle_{\omega} \\
& \quad=\exp \left\{\frac{1}{2} \int_{0}^{\beta} d \lambda \int_{0}^{\beta} d \lambda^{\prime} G_{\omega}\left(\lambda-\lambda^{\prime}\right) f(\lambda) f^{*}\left(\lambda^{\prime}\right)\right\},
\end{aligned}
$$

we readily write

$$
\lim _{\beta \rightarrow \infty}\left\langle e^{i \vec{Q} \cdot\left[\vec{r}_{1}(\lambda)-\vec{r}_{1}\left(\lambda^{\prime}\right)\right]}\right\rangle_{\mathcal{S}_{0}}=e^{-q^{2} D_{1,1}\left(\lambda-\lambda^{\prime}, 0\right)} e^{-q_{z}^{2} D_{1,1}\left(\lambda-\lambda^{\prime}, \Omega\right)},
$$

$$
\begin{aligned}
& \lim _{\beta \rightarrow \infty}\left\langle e^{i \vec{Q} \cdot\left[\vec{r}_{1}(\lambda)-\vec{r}_{2}\left(\lambda^{\prime}\right)-\vec{a}\right]}\right\rangle_{\mathcal{S}_{0}} \\
& \quad=e^{-q^{2} D_{1,2}\left(\lambda-\lambda^{\prime}, 0\right)} e^{-q_{z}^{2} D_{1,2}\left(\lambda-\lambda^{\prime}, \Omega\right)},
\end{aligned}
$$

where

$$
\begin{aligned}
D_{1,1}(\tau, \Omega) & =\frac{1}{4} \sum_{i=0}^{3} \lambda_{i}^{2}(\Omega)\left(1-e^{-\xi_{i}(\Omega)|\tau|}\right), \\
D_{1,2}(\tau, \Omega)= & \frac{1}{4} \sum_{i=0,1} \lambda_{i}^{2}(\Omega)\left(1-e^{-\xi_{i}(\Omega)|\tau|}\right) \\
& +\frac{1}{4} \sum_{i=2,3} \lambda_{i}^{2}(\Omega)\left(1+e^{-\xi_{i}(\Omega)|\tau|}\right) .
\end{aligned}
$$

Using the integral transform

$$
\int_{0}^{\beta} d \lambda \int_{0}^{\beta} d \lambda^{\prime} F\left(\left|\lambda-\lambda^{\prime}\right|\right)=2 \beta \int_{0}^{\beta / 2} d \tau F(\tau),
$$

valid for $F(\beta-\tau)=F(\tau)$, we can express the variational upper bound to the ground state energy of the "two-electron + phonon" complex in the following convenient form

$$
\begin{aligned}
E_{g}= & -3 w+\sum_{n=1,2} \frac{1}{n} \sum_{i=0}^{3} \xi_{i}\left(\Omega_{n}\right)+K \sum_{n=1,2} \frac{2}{n} D_{1,2}\left(0, \Omega_{n}\right) \\
& +U \sum_{Q} \frac{4 \pi}{Q^{2}} e^{i \vec{Q} \cdot \vec{a}} e^{-q^{2} D_{1,2}\left(0, \Omega_{1}\right)} e^{-q_{z}^{2} D_{1,2}\left(0, \Omega_{2}\right)} \\
& -4 \int_{0}^{\infty} d \tau e^{-w \tau} \sum_{n=1,2} \frac{2}{n}\left\{c_{s} D_{1,1}\left(\tau, \Omega_{n}\right)+c_{m} D_{1,2}\left(\tau, \Omega_{n}\right)\right\} \\
& -2 \sum_{Q} V_{Q}^{2} \int_{0}^{\infty} d \tau e^{-\tau\left\{e^{-q^{2} D_{1,1}\left(\tau, \Omega_{1}\right)} e^{-q_{z}^{2} D_{1,1}\left(\tau, \Omega_{2}\right)}\right.} \\
& \left.+e^{i \vec{Q} \cdot \vec{a}} e^{-q^{2} D_{1,2}\left(\tau, \Omega_{1}\right)} e^{-q_{z}^{2} D_{1,2}\left(\tau, \Omega_{2}\right)}\right\} .
\end{aligned}
$$

Here, for notational convenience, we have introduced

$$
\Omega_{n}= \begin{cases}0 & \text { for } n=1, \\ \Omega & \text { for } n=2 .\end{cases}
$$

Using Eqs. (21) and (32), the variational parameters $K, c_{s}$ and $c_{m}$ can be expressed in terms of $w$ and $\omega_{i}(i=1,2,3)$,

$$
K=\frac{1}{2}\left(\omega_{1}^{2}-\omega_{2}^{2}-\omega_{3}^{2}\right),
$$

$$
\left\{\begin{array}{l}
c_{s} \\
c_{m}
\end{array}\right\}=\frac{1}{8 w}\left\{w^{2}\left(\omega_{1}^{2}-w^{2}\right) \pm\left(\omega_{2}^{2}-w^{2}\right)\left(w^{2}-\omega_{3}^{2}\right)\right\} \text {. }
$$

Consequently, one can treat $w$ and $\left\{\omega_{i}\right\}$ as an alternative set of variational parameters satisfying the intrinsic relation

$$
0 \leqslant \omega_{3} \leqslant w \leqslant \omega_{2} \leqslant \omega_{1} \text {. }
$$


When the two electrons are set a large distance apart, i.e., in the limit $|\vec{a}| \rightarrow \infty$, the energy expression (44) should conform exactly to that of a single polaron multiplied by a factor of 2 . Indeed, a careful examination reveals that in this extreme, $\omega_{3} \rightarrow 0, \omega_{1} \rightarrow \omega_{2}$, and consequently, the present results reduce to those reported previously by Senger and Erçelebi ${ }^{30}$ for the one-polaron problem with an identical framework consisting of the same parabolic confining potential (see Appendix A). We should further emphasize that energetically the most favorable bipolaronic state has been found ${ }^{14}$ to take over for $\vec{a}=0$. Therefore, in the foregoing numerical calculations and discussions, we shall presume a vanishing mean separation between the electrons.

With the corresponding values of $K, c_{s}$ and $c_{m}$, Eq. (46), substituted in Eq. (44) and having projected out the $\vec{Q}$ summations, the variational bipolaron energy can be written as a function of $w$ and $\omega_{i}(i=1,2,3)$, given by

$$
\begin{aligned}
E_{g}= & -3 w+\sum_{n=1,2} \frac{1}{n} \sum_{i=0}^{3} \xi_{i}\left(\Omega_{n}\right) \\
& +\frac{U}{\sqrt{\pi D_{1,2}\left(0, \Omega_{2}\right)}} F\left(\frac{D_{1,2}\left(0, \Omega_{1}\right)}{D_{1,2}\left(0, \Omega_{2}\right)}\right) \\
& -\sum_{n=1,2} \frac{1}{2 n}\left\{\frac{\omega_{1}^{2}-w^{2}}{\xi_{0}\left(\Omega_{n}\right)+\xi_{1}\left(\Omega_{n}\right)}+\frac{\omega_{2}^{2}+\omega_{3}^{2}-w^{2}}{\xi_{2}\left(\Omega_{n}\right)+\xi_{3}\left(\Omega_{n}\right)}\right. \\
& \left.+\frac{\omega_{2}^{2} \omega_{3}^{2}}{\xi_{2}\left(\Omega_{n}\right) \xi_{3}\left(\Omega_{n}\right)\left[\xi_{2}\left(\Omega_{n}\right)+\xi_{3}\left(\Omega_{n}\right)\right]}\right\} \\
& -\alpha \sqrt{\frac{2}{\pi}} \int_{0}^{\infty} d \tau e^{-\tau}\left\{\frac{1}{\sqrt{D_{1,1}\left(\tau, \Omega_{2}\right)}} F\left(\frac{D_{1,1}\left(\tau, \Omega_{1}\right)}{D_{1,1}\left(\tau, \Omega_{2}\right)}\right)\right. \\
& \left.+\frac{1}{\sqrt{D_{1,2}\left(\tau, \Omega_{2}\right)}} F\left(\frac{D_{1,2}\left(\tau, \Omega_{1}\right)}{D_{1,2}\left(\tau, \Omega_{2}\right)}\right)\right\},
\end{aligned}
$$

where $\Omega_{1}$ and $\Omega_{2}$ have to be accounted for having values zero and $\Omega$, respectively, and

$$
F(x)=\frac{\arctan (\sqrt{x-1})}{\sqrt{x-1}} .
$$

\section{Integer-dimensional-space limits}

Before we present our results at large, we find it useful to investigate the conformity with the extreme limits of the bulk and strict two-dimensional cases which have already been studied extensively in the literature. When $\Omega$ is set equal to zero, the present model readily conforms to that tackled in a previous paper by Verbist, Peeters, and Devreese, ${ }^{14}$ and duplicates the same results presented therein for the bulk bipolaron. In this limit the parameters $\Omega_{n}$ Eq. (45), become both zero leading to the simplification: $\mathrm{F}(x)=1$. Using further Eq. (31) in Eq. (48), the 3D ground state energy can be written in the simple form, given by

$$
\begin{aligned}
E_{g}^{(3 \mathrm{D})}= & -N w+\frac{N}{2}\left(\omega_{1}+\omega_{2}+\omega_{3}\right)+C_{1} \frac{U}{\sqrt{D_{1,2}(0,0)}} \\
& -\frac{N}{4}\left\{\frac{\omega_{1}^{2}-w^{2}}{\omega_{1}}+\frac{\omega_{2}^{2}+\omega_{2} \omega_{3}+\omega_{3}^{2}-w^{2}}{\omega_{2}+\omega_{3}}\right\} \\
& -\alpha C_{2} \int_{0}^{\infty} d \tau e^{-\tau}\left\{\frac{1}{\sqrt{D_{1,1}(\tau, 0)}}+\frac{1}{\sqrt{D_{1,2}(\tau, 0)}}\right\}
\end{aligned}
$$

in which $N=3, C_{1}=\pi^{-1 / 2}$, and $C_{2}=(2 / \pi)^{1 / 2}$.

Going over to the strict $2 \mathrm{D}$ characterization of the polarons, and recalling that $\Omega_{2}$ stands for $\Omega$, whereas $\Omega_{1}=0$, we have the set of eigenfrequencies (29), (30), relevant to the $z$ axis, to simplify as

$$
\lim _{\Omega_{2} \rightarrow \infty} \xi_{i}\left(\Omega_{2}\right)= \begin{cases}w & \text { for } i=0 \text { and } 3, \\ \infty & \text { for } i=1 \text { and } 2 .\end{cases}
$$

Moreover, using Eqs. (27), (28), and (34), we further have the corresponding $\lambda_{i}(i=0,1,2,3)$, all to vanish. It thus follows that the functions $D_{1,1}\left(\tau, \Omega_{2}\right)$ and $D_{1,2}\left(\tau, \Omega_{2}\right)$, Eqs. (41), (42), both reduce to zero. Hence, evaluating

$$
\frac{1}{\sqrt{D_{1, i}\left(\tau, \Omega_{2}\right)}} \mathrm{F}\left(\frac{D_{1, i}\left(\tau, \Omega_{1}\right)}{D_{1, i}\left(\tau, \Omega_{2}\right)}\right) \rightarrow \frac{\pi}{2 \sqrt{D_{1, i}\left(\tau, \Omega_{1}\right)}},
$$

we obtain the corresponding ground state energy in two dimensions to have exactly the same form (50) as for the bulk case, except that we now have $N=2, C_{1}=\sqrt{\pi} / 2$, and $C_{2}$ $=(\pi / 2)^{1 / 2}$.

\section{RESULTS AND CONCLUSION}

Since analytic minimization of $E_{g}(48)$ is not possible, the determination of the optimal fits to the variational parameters $w$ and $\left\{\omega_{i}\right\}(i=1,2,3)$ requires numerical treatment. In our computations we shall trace the domain of stability $\{\eta$ $\left\langle\eta_{c}, \alpha>\alpha_{c}\right\}$ of the bipolaron as a function of the confining parameter $\Omega$.

The criterion for which a stable bipolaron state takes place will be derived by demanding that the ground state energy of the pair of composite polarons making up the bipolaron be lower than twice the energy of one single polaron. To provide a consistent comparison of the variational energy minima of the bipolaron system with those of the single polaron case, one needs further to compute the corresponding one-polaron energy values $E_{g}^{(1)}$ derived within an identical framework of the present model and formalism, and under the same numerical precision. On this purpose, one may either carry out a parallel variational computation of Eq. (44) in the limit $a \rightarrow \infty$ (see Appendix A), or alternatively, refer directly to the series of equations (13), (22), (25)-(30) given in a preceding paper ${ }^{30}$ pertaining to the study of the Feynman one-polaron problem consisting of the same quadratic confinement potential.

In exploiting the variational bipolaron energy one faces an entangled admixture of a series of competitive aspects introduced by the parameters $\alpha, \eta$, and $\Omega$ which characterize the system. It should be evident that these parameters do not 
enter the problem in an independent way but all together take part in the binding in connected and somewhat involved manners, opposing the effect of one against the other. Distinguished from the case of a three- or two-dimensional bipolaron, the extra parameter $\Omega$ adds more to the complexity in the delicate balance between the Coulomb and phonon counterparts of the problem and their percentual involvements in the binding. Clearly, in a confined volume where $\Omega$ is tuned from zero to large values, i.e., when the particles are squeezed to get closer, one expects their kinetic energy to increase and the Coulomb repulsion between them to become stronger. In the meantime, due to the rapid charge density fluctuations of the pair of electrons, the phonon-coupling becomes pseudoenhanced leading to a more effective and deeper "phonon-mediated" interaction between the particles to oppose and counterbalance the kinetic and Coulomb repulsions. Thus, the overall role of the confinement on the phonon-coupling induced localization of the electronelectron pair and the withstanding repulsion is to make these competing counter aspects stronger. What is more peculiar to the present context is that during when $\Omega$ is varied, the phonon-coupling and the Coulomb strengths may not in general grow or decrease monotonically in concert at the same rate and consequently, the relative dominating strength of either the Coulomb potential or the electron-phonon interaction over the other may become altered as a function of the degree of confinement. Yet, a further aspect of the problem intruded by the confining potential is that, in the Q2D geometry, the electrons are confined along only one spatial coordinate, but are free to expand and relax themselves in the transverse directions normal to the confining barriers; thus, in the overall, resulting in a comparatively increased interparticle separation and a relatively weakened repulsive interaction against the lattice polarization field which holds the particles together. It is therefore the mutual competition between such aforementioned aspects of the problem and the interrelated roles which the parameters $U, \alpha$, and $\Omega$ play all together that lead to the formation (or dissociation) of a bipolaron.

The common theoretical prediction led by the relevant works in the literature is that bipolaron formation is more favorable in 2D than it is in bulk. For instance, with parameter $\eta$ set equal to zero, i.e., when the Coulomb repulsion is thought of as tuned down to its hypothetical minimum strength $(U=\sqrt{2} \alpha)$, the critical value of the coupling constant over which the bipolaron state can form is found to be $\left.\alpha_{c}^{(3 \mathrm{D})}\right|_{\eta=0}=6.85$ and $\left.\alpha_{c}^{(2 \mathrm{D})}\right|_{\eta=0}=2.90$ in three and two dimensions, respectively. ${ }^{14}$ For actual material parameters of interest where $\eta>0$, the corresponding critical coupling constants scale inevitably to larger values so as to compete with the stronger Coulomb repulsion, and yet, regardless of $\eta$, one always has $\alpha_{c}^{(2 \mathrm{D})}$ to lie considerably deviated below $\alpha_{c}^{(3 \mathrm{D})}$ due to that the electrons interact more effectively with the phonons in two dimensions, and consequently, a pseudoenhanced effective electron-phonon interaction leads to a relatively smaller numerical value of the coupling constant. Such a conspicuous feature met in switching the dimensionality from three to two should naturally lead one to await that, in the quasi-two-dimensional (Q2D) configuration where $\Omega$ is tuned from zero to large values, an increased degree of confinement should play a constructive role in fa-

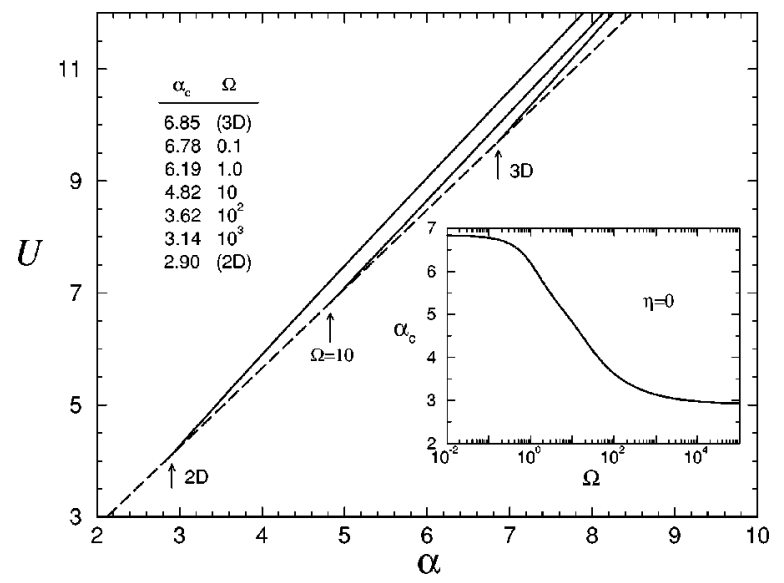

FIG. 1. The phase diagram for bipolaron formation in the space of the Coulomb coefficient and the electron-phonon coupling constant. The narrow triangular-like region bounded from below by the dashed line and from above by the top (bottom) solid line is the phase domain for a stable bipolaron in the two- (three-) dimensional limit as generated by Verbist, Peeters, and Devreese (Refs. 11,14). The middle solid line is for the quasi-two-dimensional $(\Omega=10)$ configuration. The arrows refer to the corresponding critical values of $\alpha$. The insets display the variation of $\alpha_{c}$ as a function of the confining parameter.

vor of bipolaronic stability; hence, the larger the value of $\Omega$ is, the less is the need for strong $\alpha$. A clear description of this trait is provided by the phase diagrams in Fig. 1 where we plot the Coulomb coefficient $U$ against $\alpha$. In the phase picture the space lying below the dashed line corresponds to $\eta<0$, and is therefore unphysical. The upper space bounded from below by the solid lines plotted for three distinctive degrees of confinement $(\Omega=0, \Omega=10$, and $\Omega \rightarrow \infty)$ gives the unstable region where the polarons choose to remain separated. Thus, it is only the narrow triangular area bounded by the dashed and either of the solid lines in which the polarons can be found in a bound state forming a stable bipolaron. The vertex of each triangular region at which the dashed and solid lines join defines an infimum for the coupling constant in the $3 \mathrm{D}$, Q2D $(\Omega=10)$, and $2 \mathrm{D}$ configurations. Coupling constants larger than these critical vertex values serve for supporting the bipolaron to conserve its stability at correspondingly stronger Coulomb repulsions. The relevant numerical data of $\alpha_{c}$ for a few sample values of $\Omega$ is tabulated in the upper left inset of the figure. A more tractable display of $\alpha_{c}$ is given in the lower right inset where we plot its variation continuously as a function of $\Omega$ encompassing the bulk and the 2D limits. The overall conclusion led by the content of Fig. 1 is that the critical $\alpha$ below which a bipolaron state is unfavorable gets shifted to smaller values as the degree of confinement is increased. Also, for a given fixed value of $\alpha$, the lower the dimensionality is, the more favorably the bipolaron state can be sustained. In numerical terms, setting $\alpha=8$ for instance, we evaluate the critical upper bound for the Coulomb coefficient beyond which the bipolaron dissociates into two individual polarons as $U_{c}$ $=11.59,11.79$, and 12.17, respectively, for the 3D, Q2D $(\Omega=10)$, and $2 \mathrm{D}$ cases. In the next paragraph we review the content of Fig. 1 from an alternative viewpoint where we give an explicit and broader picture of $U_{c}$ against $\Omega$ over a 


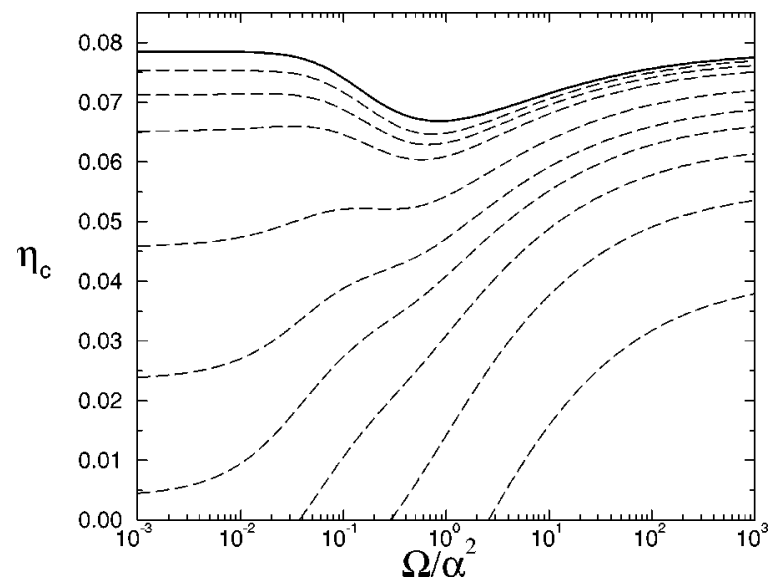

FIG. 2. The critical ratio $\eta_{c}$ as a function of degree of confinement for a succession of different $\alpha$ values. The dashed curves from bottom to top give the path-integral results obtained for $\alpha$ $=4,5,6,7,8,10,15,20,30$. The topmost solid curve is universal for all large $\alpha$ and has been obtained within the framework of the strongcoupling polaron theory.

succession of distinctive $\alpha$ values extended towards the strong-coupling limit.

Setting $\alpha$ fixed at any desired value, Eq. (5) allows us to trace the critical condition on the Coulomb strength as a function of the degree of confinement, displayed equivalently in terms of the alternative related parameter $\eta$. In Fig. 2 we plot a series of curves describing the effect of confinement on the critical value of $\eta$ for different coupling constants ranging in between 4 and 30. In the same figure, for the purpose of comparison, we also include a supplementary layout of $\eta_{c}$ versus $\Omega$ derived within the framework of the strong-coupling adiabatic polaron theory (see Appendix B). Over the scale of the abscissa we choose to express $\Omega$ in units $\alpha^{2}$, mainly for two reasons; one is that the ratio $\Omega / \alpha^{2}$ (rather than bare $\Omega$ ) proves to be a more sensible measure of the degree of confinement, and the other stems from that, in the strong coupling expansion to leading order in $\alpha$, the ground state energy is seen to be proportional to the square of the coupling constant, i.e., $E_{\mathrm{g}}=-c \alpha^{2}$, where the corresponding coefficient of proportionality bears a functional relation solely to $\Omega / \alpha^{2}$ for both the single-polaron and twopolaron systems. ${ }^{26}$ We should note that the polarons can feel the boundary potential and enter a regime of reduced dimensionality only when the effective well width $(\sim 1 / \sqrt{\Omega})$ is smaller than, or at least comparable with the mean (bi)polaron size $(\sim 1 / \alpha)$, and consequently, even a large $\Omega$ would not mean anything, but bulk medium, unless $\alpha<\sqrt{\Omega}$. It is therefore due to this reasoning that we are tempted to accept the ratio $\Omega / \alpha^{2}$ as a convenient measure of confinement. On the other hand, a careful examination of Eqs. (1) and (2) reveals that, if the energies are scaled by $\alpha^{2}$ and lengths by $\alpha$, the only modification in the Hamiltonian would be to replace the confining parameter $\Omega$ by $\Omega / \alpha^{2}$ and the Coulomb coefficient $U$ by $U / \alpha(=1 /[1-\eta])$. Thus, at "strong coupling," if $\eta_{c}$ is plotted against $\Omega / \alpha^{2}$, rather than $\Omega$, we find that one can display the phase boundary on one universal curve with no loss in generality for all large $\alpha$.

A plain implication led by the plots in Fig. 2 is that, the stronger the phonon coupling, or the larger the degree of confinement is, the more easily the bipolaron state can form and be supported against the repulsive Coulomb potential. We should remark that any plot of $\eta_{c}$ pertaining to $\alpha$ $<6.85$ intersects the abscissa at a nonzero value of $\Omega$; hence, for not strong enough $\alpha$, a bipolaron can form only beyond a critical degree of confinement, and the larger the coupling constant is, the smaller is the corresponding critical $\Omega$. Clearly, for $\alpha \geqslant 6.85$ one has $\Omega_{c}=0$, where in this case bipolaron formation is favorable even in bulk, and the sole effect of the confinement is to take over in favor of enhancing the already established stability of the bipolaron.

A careful look at the series of curves from bottom to top reveals that as $\alpha$ is made indefinitely large, a peculiar feature starts to build up, and contrary to our anticipations, we observe that the critical $\eta$ does no more display a steady increasing behavior interpolating between the 3D and 2D limits, but instead attains its corresponding 2D value after having gone through a minimum located at some place about $\Omega / \alpha^{2} \simeq 1$. We feel that this salient characteristic is an implicit consequence of the dominating effect of either the Coulomb repulsion or the phonon mediated attraction over the other, and the cross overing of the competition between these counter aspects as the confining parameter is varied. In regard with the strong-coupling limit, we should add the note that, as $\alpha$ is adjusted to larger and larger values, the sequence of dashed (path-integral) curves converge towards the topmost solid curve which we have obtained independently adopting the adiabatic approximation, the details of which we summarize in Appendix B. In the extreme strongcoupling limit we obtain $\eta_{c}=0.079$ in both three and two dimensions, as reported earlier in Ref. 16.

Before we close our discussions we would like to shed some insight into polaron-polaron versus bipolaron bound state energies and display the profiles of the corresponding variational parameters $w$ and $\left\{\omega_{i}\right\}$ against the degree of confinement. To provide an explicit track of the evolution of the "polaron-polaron" complex as a function of the effective dimensionality, we refer back to the simple case for which we artificially set $\eta=0$, and choose $\alpha=5$ as a sample value being somewhat smaller than the corresponding bulk critical minimum, $\left.\alpha_{c}^{(3 \mathrm{D})}\right|_{\eta=0}=6.85$. In Fig. 3(a) we plot the possible bipolaronic binding energy

$$
E_{b}=\Omega-E_{g}
$$

accompanied by twice the binding energy of the corresponding single polaron over the range $0 \leqslant \Omega \leqslant 10$. To give a complementary understanding of the polaron-polaron (PP) and bipolaron (BP) phases and the transition from one phase to the other, we also display the variational parameters as a function of $\Omega$ [see Fig. 3(b)]. We at first note that for $\Omega$ $>7.21$, i.e., in the region " $\mathrm{BP}(\mathrm{PP})$," the bipolaronic phase is energetically more likely to show up compared to the PP phase of two individual polarons, and yet, increasing the degree of confinement enhances the stability of the BP phase. For $\Omega<7.21$, however, the state of two individual polarons is favored. In the region " $\mathrm{PP}(\mathrm{BP})$, ," $(4.53<\Omega$ $<7.21)$ the bipolaronic phase is seen to persist rather recessively where, with decreasing $\Omega$, the corresponding local minimum in its ground state energy starts to lose its depth and eventually diminishes for $\Omega=4.53$. When $\Omega$ is made 


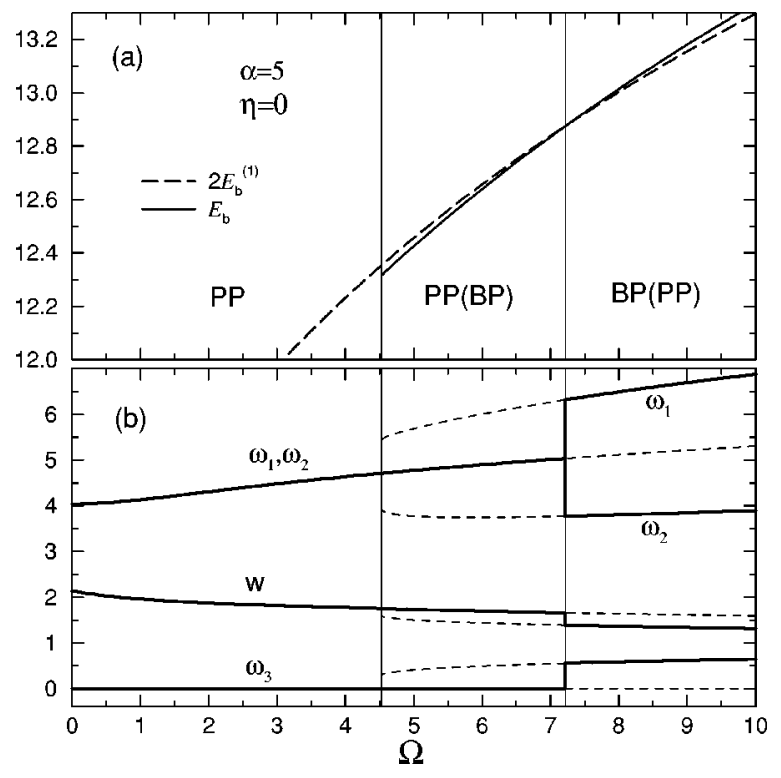

FIG. 3. (a) Bipolaron and two single-polaron binding energies and (b) the variational parameters, as functions of the degree of confinement.

even smaller, i.e., as the bulk limit is approached, one achieves solely the PP phase with whatsoever no relevance to any possible bipolaronic characterization of the twopolaron system. In Fig. 3(b) the variational parameters pertaining to the energetically favorable phase are plotted in boldface curves and those which correspond to the metastable state of either phase are given in dashed lines. It should be noted that if the bipolaronic phase should indeed be realized either as a metastable state or otherwise, the corresponding variational parameters all achieve nonzero and distinctive values. The phase of two independent polarons, however, is characterized by that $\omega_{1}$ and $\omega_{2}$ coalesce into one single curve, and $\omega_{3}=0$ regardless of $\Omega$. Consequently, one has $w$ and $\nu\left(=\omega_{1}=\omega_{2}\right)$ to be recognized as the well established Feynman variational parameters of the usual onepolaron problem. ${ }^{27,30}$

\section{ACKNOWLEDGMENT}

R. T. Senger acknowledges the financial support of the Münir Birsel Foundation TUBITAK.

\section{APPENDIX A: ONE-POLARON LIMIT}

When the electrons are thought of as infinitely separated, the part of the trial action relevant to the Coulomb and mutual interactions must fall off to zero, and from Eqs. (16) and (18) it is not very difficult to guess that the variational coefficients $K$ and $c_{m}$ should both tend to zero. From Eq. (46), we find that the only way this can happen is to have $\omega_{3}$ $\rightarrow 0$ and $\omega_{1}-\omega_{2} \rightarrow 0$. Hence, setting $\nu=\omega_{1}=\omega_{2}$, we further have the self-energy coefficient to simplify to

$$
c_{s}=\frac{1}{4} w\left(\nu^{2}-w^{2}\right)
$$

which we identify exactly as the coefficient in the energy term given by Eq. (27) in a previous paper on the confined one-polaron problem by Senger and Erçelebi, ${ }^{30}$ which hereafter will be referred to as SE.

A further simplification is that the eigenfrequencies $\xi_{i}(\Omega)$ (29), (30) now read as

$$
\begin{aligned}
& \xi_{0}(\Omega)=\xi_{3}(\Omega) \rightarrow \xi_{1}^{(\mathrm{SE})}(\Omega), \\
& \xi_{1}(\Omega)=\xi_{2}(\Omega) \rightarrow \xi_{2}^{(\mathrm{SE})}(\Omega),
\end{aligned}
$$

where $\xi_{1}^{(\mathrm{SE})}(\Omega)$ and $\xi_{2}^{(\mathrm{SE})}(\Omega)$ are the eigenfrequencies given by Eq. (19) in SE. We should also remark that in the limit $a \rightarrow \infty$, the rapidly oscillating term $e^{i \vec{Q} \cdot \vec{a}}$ involved in Eq. (44) yields zero mean value.

In view of all the above simplifications, we rewrite the energy expression (44) as

$$
\begin{aligned}
\lim _{a \rightarrow \infty} E_{g}= & \sum_{n=1,2} \frac{2}{n}\left[\xi_{0}\left(\Omega_{n}\right)+\xi_{1}\left(\Omega_{n}\right)-w\right] \\
& -4 c_{s} \int_{0}^{\infty} d \tau e^{-w \tau}\left[2 D_{1,1}\left(\tau, \Omega_{1}\right)+D_{1,1}\left(\tau, \Omega_{2}\right)\right] \\
& -2 \sum_{Q} V_{Q}^{2} \int_{0}^{\infty} d \tau e^{-\tau} \exp \left\{-q^{2} D_{1,1}\left(\tau, \Omega_{1}\right)\right. \\
& \left.-q_{z}^{2} D_{1,1}\left(\tau, \Omega_{2}\right)\right\}
\end{aligned}
$$

which indeed is a replica of twice the expression for the one-polaron ground state energy given through the series of equations (13), (22), (25)-(30) in SE. The correspondence with the notation of SE can be established through

$$
D_{1,1}\left(\tau, \Omega_{i}\right)=\frac{\tau}{2 \sigma_{i}(\tau)},
$$

where $\sigma_{i}(\tau)$ is defined by SE Eq. (28).

\section{APPENDIX B: STRONG-COUPLING APPROXIMATION}

In the $\alpha \gg 1$ limit, an alternative approach is the conventional Pekar adiabatic theory ${ }^{31}$ which imposes a product Ansatz separable in the particle and phonon coordinates, i.e.,

$$
\Psi=\Phi(\vec{R}, \vec{r}) \times \exp \sum_{Q} s_{Q}\left(a_{Q}-a_{Q}^{\dagger}\right)|0\rangle
$$

In the above, the exponential operator acting on the phonon vacuum is the displaced oscillator transformation where $s_{Q}$ is a variational parameter determined from the requirement $\partial\langle\Psi|\mathrm{H}| \Psi\rangle / \partial s_{Q}=0$ to yield

$$
s_{Q}=V_{Q} \sum_{j=1,2}\left\langle\Phi\left|e^{ \pm i \vec{Q} \cdot \vec{r}_{j}}\right| \Phi\right\rangle .
$$

For the particle part of the trial state, we assume variational oscillator-type wave functions separable in the center of mass, $\vec{R}=\left(\vec{r}_{1}+\vec{r}_{2}\right) / 2$ and the relative $\vec{r}=\vec{r}_{1}-\vec{r}_{2}$ coordinates, i.e., 


$$
\begin{aligned}
\Phi(\vec{R}, \vec{r})= & N \exp \left\{-\frac{1}{2} \lambda_{1}^{2}\left(R_{\rho}^{2}+\mu_{1}^{2} R_{z}^{2}\right)\right\} \\
& \times \exp \left\{-\frac{1}{2} \lambda_{2}^{2}\left(r_{\rho}^{2}+\mu_{2}^{2} r_{z}^{2}\right)\right\}
\end{aligned}
$$

in which $\vec{R}_{\rho}$ and $R_{z}$ stand for the lateral and $z$ components of the center of mass position vector, and the components $\vec{r}_{\rho}$ and $r_{z}$ have similar meanings for the relative position vector. $N$ is the normalization constant.

The bipolaron ground state energy can then be obtained through a numerical minimization of $E_{g}=\langle\Psi|\mathrm{H}| \Psi\rangle$ with respect to the set of four variational parameters $\left\{\lambda_{i}, \mu_{i}\right\}$ (i $=1,2)$. In computing the critical phase boundary we have made correspondence with the results provided in a preceding paper $^{32}$ pertaining to the strong-coupling study of the one-polaron problem consisting of the same quadratic confinement potential.
${ }^{1}$ H. Haken, in Polarons and Excitons, edited by C.G. Kuper and G.D. Whitfield (Plenum, New York, 1963).

${ }^{2}$ S.D. Mahanti and C.M. Varma, Phys. Rev. B 6, 2209 (1972).

${ }^{3}$ J. Sak, Phys. Rev. B 6, 2226 (1972).

${ }^{4}$ S.P. Kuleshov, V.A. Matveev, and M.A. Smondyrev (unpublished).

${ }^{5}$ E.A. Kochetov, S.P. Kuleshov, V.A. Matveev, and M.A. Smondyrev, Theor. Math. Phys. 30, 117 (1978)

${ }^{6}$ M.F. Bishop and A.W. Overhauser, Phys. Rev. B 23, 3627 (1981).

${ }^{7}$ Y. Takada, Phys. Rev. B 26, 1223 (1982).

${ }^{8}$ H. Hiramoto and Y. Toyozawa, J. Phys. Soc. Jpn. 54, 245 (1985).

${ }^{9}$ J. Adamowski, Phys. Rev. B 39, 3649 (1989).

${ }^{10}$ T.K. Mitra, Phys. Lett. A 142, 398 (1989).

${ }^{11}$ G. Verbist, F.M. Peeters, and J.T. Devreese, Solid State Commun. 76, 1005 (1990).

${ }^{12}$ V. Cataudella, G. Iadonisi, and D. Ninno, Phys. Scr. T39, 71 (1991).

${ }^{13}$ F. Bassani, M. Geddo, G. Iadonisi, and D. Ninno, Phys. Rev. B 43, 5296 (1991).

${ }^{14}$ G. Verbist, F.M. Peeters, and J.T. Devreese, Phys. Rev. B 43, 2712 (1991).

${ }^{15}$ S. Sil, A.K. Giri, and A. Chatterjee, Phys. Rev. B 43, 12642 (1991).

${ }^{16}$ G. Verbist, M.A. Smondyrev, F.M. Peeters, and J.T. Devreese, Phys. Rev. B 45, 5262 (1992).

${ }^{17}$ J. Adamowski and S. Bednarek, J. Phys.: Condens. Matter 4, 2845 (1992).
${ }^{18}$ A. Chatterjee and S. Sil, Int. J. Mod. Phys. B 7, 4763 (1993).

${ }^{19}$ V.M. Fomin and M.A. Smondyrev, Phys. Rev. B 49, 12748 (1994).

${ }^{20}$ C. Qinghu, W. Kelin, and W. Shaolong, Phys. Rev. B 50, 164 (1994).

${ }^{21}$ F. Luczak, F. Brosens, and J.T. Devreese, Phys. Rev. B 52, 12 743 (1995).

${ }^{22}$ M.A. Smondyrev, J.T. Devreese, and F.M. Peeters, Phys. Rev. B 51, 15008 (1995).

${ }^{23}$ S. Sahoo, J. Phys.: Condens. Matter 7, 4457 (1995).

${ }^{24}$ M.A. Smondyrev and J.T. Devreese, Phys. Rev. B 53, 11878 (1996).

${ }^{25}$ S. Mukhopadhyay and A. Chatterjee, J. Phys.: Condens. Matter 8, 4017 (1996).

${ }^{26}$ R.T. Senger and A. Erçelebi (unpublished).

${ }^{27}$ R.P. Feynman, Phys. Rev. 97, 660 (1955).

${ }^{28}$ D.M. Larsen, Proceedings of the 17th International Conference on the Physics of Semiconductors, edited by J.D. Chadi and W. Harrison (Springer, Berlin, 1985), p. 421.

${ }^{29}$ S.N. Klimin, E.P. Pokatilov, and V.M. Fomin, Phys. Status Solidi B 184, 373 (1994).

${ }^{30}$ R.T. Senger and A. Erçelebi, J. Phys.: Condens. Matter 9, 5067 (1997).

${ }^{31}$ S.I. Pekar, Untersuchungen über die Elektronentheorie der Kristalle (Akademie, Berlin, 1954).

${ }^{32}$ T. Yıldırım and A. Erçelebi, J. Phys.: Condens. Matter 3, 1271 (1991). 